\title{
A nurse-based intervention for improving medication adherence in cardiovascular patients: an evaluation of a randomized controlled trial
}

This article was published in the following Dove Press journal: Patient Preference and Adherence

\author{
Angelien Sieben' \\ Hein AW van Onzenoort ${ }^{2,3}$ \\ Sandra van Dulmen ${ }^{4-6}$ \\ CJHM van Laarhoven ${ }^{7}$ \\ Sebastian JH Bredie ${ }^{8}$ \\ 'Department of Surgery, Division of \\ Vascular Surgery, Radboud University \\ Nijmegen Medical Center, Nijmegen, the \\ Netherlands; ${ }^{2}$ Department of Clinical \\ Pharmacy, Amphia Hospital, Breda, the \\ Netherlands; ${ }^{3}$ Department of Clinical \\ Pharmacy and Toxicology, Maastricht \\ University Medical Center+, Maastricht, \\ the Netherlands; ${ }^{4}$ Department of \\ Primary and Community Care, Radboud \\ University Medical Center, Nijmegen, the \\ Netherlands; ${ }^{5}$ NIVEL (Netherlands insti- \\ tute for health services research), \\ Utrecht, the Netherlands; ${ }^{6}$ Faculty of \\ Health and Social Sciences, University of \\ South-Eastern Norway, Drammen, \\ Norway; ${ }^{7}$ Department of General \\ Surgery, Radboud University Nijmegen \\ Medical Center, Nijmegen, the \\ Netherlands; ${ }^{8}$ Department of General \\ Internal Medicine, Division of Vascular \\ Medicine, Radboud University Medical \\ Center, Nijmegen, the Netherlands
}

Correspondence: Angelien Sieben Radboud University Nijmegen Medical Center, Geert Grooteplein I0, Postbus 9101 , Nijmegen $6500 \mathrm{HB}$, the

Netherlands

Tel +3I 2436168234

Email angelien.sieben@radboudumc.n
Background: Poor medication adherence is a limitation in the secondary prevention of cardiovascular diseases (CVDs) and leads to increased morbidity, mortality, and costs.

Purpose: To examine the process and effect of a nurse-led, web-based intervention based on behavioral change strategies to improve medication adherence in patients with CVD.

Patients and methods: In this single-center, prospective, controlled clinical trial, cardiovascular patients were assigned to usual care, usual care plus a personalized website, or usual care plus a personalized website and personal consultations. Primary outcome was the level of adherence to cardiovascular medication. Data collection occurred between October 2011 and January 2015.

Results: In total, 419 patients were randomized. Just 77 patients logged on the website and half of the invited patients attended the group consultation. Due to the limited use of the website, we combined the results of usual care and the usual care plus website group in one group (usual care) and compared these with the results of the group which received the nurse intervention (intervention group). No significant difference in adherence between the usual care group and the intervention group was observed. The adherence level in the usual care group was $93 \%$, compared to $89 \%$ in the intervention group ( $p=0.08$ ). $29 \%$ (usual care) and $31 \%$ (intervention group) of the patients showed a low adherence according to the Modified Morisky Scale $\AA$ ( $p$-value=0.94). The mean necessity concern differential was 3.8 with no differences between the two studied groups (mean 3.8 vs mean $3.9, p$-value $=0.86$ ).

Conclusion: Our intervention program did not show an effect. This could indicate that structured usual care provided to all cardiovascular patients already results in high medication adherence or that shortly after a cardiovascular event adherence is high. It could also indicate that the program did not have enough impact because there was not enough compliance with the intervention protocol.

Trial registration: ID number NCT01449695, approved May 2011.

Keywords: medication adherence, nurses, e-health, Health Belief Model, cardiovascular

\section{Introduction}

Cardiovascular disease (CVD) is the leading cause of death worldwide. Although lifestyle risk factors of patients with high cardiovascular risk are monitored regularly as part of the risk-reducing programs, there is limited structural attention to medication adherence. ${ }^{1}$ This is startling because it is known that poor medication adherence is a major limitation in the secondary prevention of CVDs that may lead to increased morbidity, mortality, and costs. ${ }^{2-4}$ Approximately $50 \%$ of the patients 
using medication for a chronic disorder do not adhere to the prescribed drug regimen according to WHO. ${ }^{5}$ In patients with CVD, adherence rates remain low, ranging between 50 and $79 \%{ }^{2,6}$ As a consequence, in Europe an estimated $9 \%$ of preventable CVD events can be attributed to poor adherence to vascular medications alone. ${ }^{7}$

Current methods for improving adherence are mostly complex and not very effective, and simple interventions that are feasible in usual practice settings are preferred. ${ }^{8}$ There is, however, a need for more understanding in patientrelated determinants of poor adherence to medication. These determinants can either be intentionally or unintentionally driven. Unintentional poor adherence occurs when patients are inclined to adhere but are not able to because of a lack of capacity or resources. Intentional poor adherence occurs when patients decide not to follow the agreed recommendations. ${ }^{9}$ To be effective, interventions should address both unintentional and intentional determinants of poor adherence. ${ }^{10}$ Central to patients' medication adherence is their judgment of their personal need for taking medication. Key beliefs that influence patients' judgment about their medication are perception of personal needs for treatment (necessity beliefs) and concerns about several potential negative consequences (concern beliefs). ${ }^{9,11,12}$ Though lifelong adherence is important in CVD, absence of symptoms in the years after an event may result in the perception that the illness is benign. This may lead to doubts about the necessity of continuous treatment. ${ }^{13}$ A patient-centered approach with emphasis on patients' perspectives might encourage CVD patients to take their medication. ${ }^{14,15}$ The principles of the patients' perspective were used in the development of the intervention under study. The intervention was, therefore, based on the Health Belief Model (HBM) ${ }^{16,17}$ in which the necessity-concern framework was applied ${ }^{9}$ and adapted for the specific purpose of this trial.

\section{Material and methods}

\section{Aim}

This study aimed to evaluate the process and outcomes of an intervention program consisting of a single group consultation, two individual follow-up consultations with a nurse, and access to an interactive personalized website, incorporating evidence-based determinants of poor adherent behavior in high-risk cardiovascular patients. The $\mathrm{HBM}^{18}$ was chosen as the central theoretical model for the interventions. The intervention program aims to improve patient's necessity and concern beliefs about medication, which is expected to lead to better adherent behavior in cardiovascular patients.

\section{Design/methodology}

The design of this study (with an acronym: the MIRROR trial) has been described in detail earlier. ${ }^{19}$ The MIRROR study is a single-center, prospective, three-arm randomized controlled clinical trial. Patients were randomly assigned to usual care (group I), usual care plus access to a personalized website (group II), or usual care, access to a personalized website plus a group consultation with a pharmacist and a nurse, followed by two individual nurse-led consultations at the outpatient clinic (group III).

\section{Sample}

All patients referred to the Radboud University Nijmegen Medical Center with a new diagnosis of acute coronary syndrome, myocardial infarction, peripheral arterial disease, an aneurysm of the aorta or transient ischemic attacks (TIA) or stroke over the last 6 months were included into the hospital CVD screening program. This screening program aims to identify cardiovascular risk factors and consists of screening of lifestyle (smoking, diet, and exercise), blood lipid levels, blood pressure, waist circumference, body mass index (BMI), glucose blood levels, and a family history of CVDs. If indicated, preventive therapies (medication and lifestyle interventions) are structurally initiated and followed over time. ${ }^{20}$ From this program, participants aged 18 years and older were eligible for the MIRROR trial if they were willing to stay in a 1-year follow-up period and provided signed informed consent for an intervention on medication adherence. Exclusion criteria were pregnancy reported by the patient, severe comorbidity (eg, lung cancer, end-stage heart failure), difficulties with Dutch language, or no access to a computer.

\section{Randomization}

Patients who consented to participate were randomized using block randomization stratified by department (neurology, vascular surgery, and cardiology) in a 1:1:1 ratio into one of the three groups. Randomization was blinded for the principal investigator and the primary researcher. The patient, the nurse, or the pharmacist delivering the individual consultations could be blinded to the intervention assignment in group III. 


\section{Power calculation}

The study was powered on adherence to medication expressed as mean possession ratio (MPR). We estimated that adherence levels at the start of the study would be $65 \%$ in each group with a SD of $30 \%$. We hypothesized that the intervention given in group II and the intervention given in group III would result in an increase of $10 \%$ and $20 \%$ in adherence to treatment, resulting in a mean adherence rate of $75 \%$ and $85 \%$ in group II and group III, respectively. Consequently, the estimated group size with a power of $80 \%$ and an alpha of 0.05 (two-sided) would be 200 in each group, resulting in 600 participants in total.

\section{Timeline}

The intervention period lasted 12 months. Within, on average, 6 weeks after the CVD event, baseline characteristics were collected for all patients. Follow-up outcomes were collected at 12 months after inclusion for all patients.

\section{Data collection \\ Process evaluation}

As recommended by the Medical Research Council Guidance, ${ }^{21}$ we included a process evaluation of this intervention program. A process evaluation helps to understand the relationship between how well an intervention was delivered, the different elements of an intervention, and the main outcomes of a trial. ${ }^{22,23}$ It furthermore improves the validity and interpretation of these outcomes and gives information so the intervention can be replicated. ${ }^{24}$ This intervention program was performed at an outpatient clinic for cardiovascular risk management in our academic hospital. Nurses, who deliver the individual consultations, already counsel cardiovascular patients in changing their lifestyle (eg, stop smoking and losing weight) through motivational interviewing techniques. In addressing the problem of nonadherence as a behavioral problem, the intervention program could fit really well in the existing clinical setting. There is also a broad recognition that nurses have a key role in understanding and addressing patients 'beliefs during consultations about their medication.

\section{Adherence}

The primary outcome of our study was adherence to cardiovascular medication. Adherence was based on pharmacy refill dates (PRD) of participants' filled prescriptions obtained from computerized pharmacy systems. Data were collected for prescribed cardiovascular drugs (plated aggregation inhibitors, lipid-modified agents, and antihypertensive drugs) for the period of 3 years prior to a patient's cardiovascular event and at least 1 year after cessation of the intervention of this trial. All prescription records included the Anatomic Therapeutic Chemical code (ATC), the names of the dispensed drugs, prescribed daily dose, quantity dispensed at each pharmacy fill, and the dates of the prescription fills. Adherence was reported as the MPR for all cardiovascular medication. The MPR was defined as the number of days of treatment dispensed divided by the number of days prescription refills. ${ }^{25}$ We calculated adherence levels at baseline (T1), at the end of the intervention (T2), and at 1 year after ending the intervention (T3). Patients with an adherence level of at least $80 \%$ were considered adherent, whereas patients with an adherence level lower than $80 \%$ were considered as nonadherent.

\section{Secondary outcomes Clinical responses}

According to the hospital screening program, blood was drawn from all patients to determine low-density lipoprotein (LDL) cholesterol levels. A recorded LDL cholesterol level of $20 \%$ above the baseline level during follow-up was considered as an indication of poor adherence. If patients used antihypertensive drugs, they were classified adherent if the systolic blood pressure was $<135 \mathrm{mmHg}$ after the intervention. Target blood pressure levels were set according to the European Society of Hypertension (ESH) recommendations (ie, a systolic blood pressure level of $<135 \mathrm{~mm} \mathrm{Hg}$ ). These office blood pressure measurements were performed according to the recommendations of the ESH with a validated automated device and based on a mean of four office measurements. ${ }^{26}$

\section{Patient outcomes}

All patients filled out the Modified Morisky Scale (MMS ${ }^{\circledR}$ ) and the Beliefs about Medicines Questionnaire (BMQ) at baseline and at the end of the follow-up period. The MMS ${ }^{\circledR}$ is a validated questionnaire consisting of eight items aimed at measuring adherence. ${ }^{27-29}$ Each item accounts for 0 or 1 in case questions are answered by No or Yes, respectively. These scores were divided into three levels of adherence: low adherence (sum score $<6$ ), medium adherence (sum score 6 to $<8$ ), and high adherence (sum score of 8 ). To evaluate patients' beliefs and perceptions about their medication, the BMQ was used. ${ }^{30}$ Respondents stated their degree of agreement with each individual statement about medicines on a five-point Likert scale. The necessity-concern differential (NCD) was calculated as the difference 
between necessity and concern scores and had a possible range of -20 to 20 . If the difference was positive, the patient perceived that the benefits of medication outweighed the concerns. Contrarily, if the differential was negative, the patient perceived more costs than benefits. ${ }^{13,31}$ To separate patients on the basis of their beliefs about the necessity of their medication and their concerns about taking medication, the total necessity and concern scores (5-25) were split at midpoint (thus 5-12 was considered as low and $13 \mathrm{t} / \mathrm{m} 25$ was considered as high). Patients were then classified into four different categories: accepting (high necessity and low concerns), ambivalent (high necessity and high concerns), skeptical (high concerns and low necessity), and indifferent (low concerns and low necessity). ${ }^{32-34}$ From all patients, the type of CVD (acute coronary syndrome, myocardial infarction, peripheral arterial disease, an aneurysm of the aorta, or TIA) was recorded. Also, the following baseline and clinical characteristics were collected: age, sex, level of education, employment status, and the country of origin. Whether patients were new or chronic users of cardiovascular medication was also registered. To classify patients as a chronic medication user, they had to use a plated aggregation inhibitor and/or a lipid-modified agents more than 2 months before baseline according to PRD. All other patients were classified as new users. The log-on information expressed as the number of log-ins, times, and dates on the personalized website of each patient were recorded.

\section{Ethics}

Written informed consent was collected from all patients prior to entering the study. The study protocol was approved by the local ethical committee. Approval for this study was obtained by the local ethical committee, the human-related research committee of the ArnhemNijmegen region (CMO no 2011/062), which applied criteria described in the Medical Scientific Research with People Act (WMO), the Helsinki Declaration, the Good Clinical Practice (GCP), EU Guideline GCP, Clinical trials guidelines on medicinal products, and CCMO guidelines.

\section{Data analysis}

Data were analyzed based on the intention-to-treat principle and evaluated by using SPSS, with descriptive statistics (mean, median, and SD) being determined for all variables. Differences between the patient groups were tested by performing an ANOVA test on the outcome measures. All sociodemographic and disease-related factors were included so they could be taken into account as potential confounders. ${ }^{14,35,36}$ To handle with missing data, we followed the recommendations for eHealth research and used multiple imputation techniques in SPSS. ${ }^{37}$ Multiple imputation is considered as the standard procedure for dealing with missing data. It has the advantage of incorporating auxiliary information about missing data into the analysis, thereby reducing bias and improving accuracy. ${ }^{38}$ Analysis of multiple imputated data shows greater power and efficiency than complete case analyses. Multiple imputations make better use of available data and can generate different results from simpler techniques. ${ }^{39}$ The datasets generated and analyzed during this study are not publicly available due to the Dutch privacy laws. But they are available from the corresponding author on reasonable request.

\section{Validity and reliability}

This study used instruments with their validity and reliability tested in previous studies. ${ }^{27,30,40}$ Participants were randomized using block randomization. The intervention was delivered by well-trained nurses who all had a training specific for delivering the intervention.

\section{Results}

\section{Process evaluation}

\section{Development of the intervention program}

The intervention program consisted of a nurse-based intervention providing structured information and motivational counseling, and a personalized visualization of cardiovascular risk levels on a website. The intervention was based on evidence-based behavior change theory, the $\mathrm{HBM}^{16}$ and the necessity-concern framework. ${ }^{9}$ This necessityconcern framework is a useful model for understanding and addressing both unintentional and intentional nonadherence. ${ }^{9}$ By using the BMQ, ${ }^{30}$ nurses could get insight into the necessity and concern beliefs of their patients and were able to tailor their consultation to the needs of each individual patient. The HBM is based on the understanding that a person will take health-related action (eg, being adherent to cardiovascular medication) given four main factors. The first two factors are perceived susceptibility and perceived severity, reflecting the understanding of the high personal risk and seriousness of a condition (eg, because of the cardiovascular event in the past, I am at greater risk for another cardiovascular event). The third factor is perceived benefits, aimed at the belief a negative health condition can be avoided (eg, being adherent to the cardiovascular medication can help 
to prevent another cardiovascular event). The last factor covers the perceived barriers, cue to action, and self-efficacy, aimed at the belief to have the ability to successfully undertake the recommended health action (eg, I know how to take my medication on a daily base). The group consultation with between 10 and 12 patients was chosen for delivering knowledge and understanding of the risks. Moreover, it also provided a gathering with other patients (peers) in which patients were given the possibility to discuss adherence behavior and learn from each other. In the individual consultations, the intervention was further tailored to each individual, so nurses were able to identify objectives for change of (un)intentional nonadherence (or a mix of both) and were able to address the determinants of patients' beliefs, perceptions and management of their illness and medication next to patients' skills and memory. ${ }^{8}$ To support the individual and group consultations, an interactive and personalized website was developed. On this website, patients could see their own cholesterol levels, blood pressure, and lifestyle (smoking, physical activity, and eating habits) in a risk monitor. Patients had the opportunity to ask questions by email to their nurse and enter changes in their medication. We choose a three-arm randomized controlled trial to determine if the website alone was effective or if group consultations and individual consultations had add-on effects. The participating nurses were trained in motivational interviewing and had applied this as part of the usual care program for several years. ${ }^{41}$ They received an extra training for this intervention. ${ }^{42,43}$

The website was expected to be effective on itself by creating awareness (risk monitor), providing (written) information, and tailoring of the information by the email facility. ${ }^{4,45}$ Risk communication and the feedback of clinical outcome can be provided personally and patients were encouraged to be active in handling their disease and medication. The website was connected to the hospital laboratory system to provide personal clinical results. Logging in was due to high privacy levels. Patients had to use a special personal code and had to confirm their password with a code they received from a text message. For developing the intervention program, we used the recommended behavior change strategies of the HBM. We tailored further by using the taxonomy of Abraham and Michie $^{46,47}$ and the coding manual by de Bruin to categorize the behavior change techniques that required to be enclosed in the intervention. ${ }^{46,48}$
For each of the components of HBM, the determinants, techniques, and application strategy that were developed are described in Table 1.

\section{Uptake of the intervention}

\section{Patient enrollment and inclusion}

Of a total of 1,201 patients with a cardiovascular event who enrolled the screening program, 900 were eligible to participate in this study. Of these, 481 declined to participate. In total, 419 patients were randomized into group $\mathrm{I}(\mathrm{n}=133)$, group II $(n=138)$, and group III $(n=148)$. Data collection occurred between October 2011 and January 2015. After randomization, 148 patients were invited to attend the group and individual consultations, of which 79 of these participated in the group consultation. One hundred and thirty-four and 79 of these patients visited the first and second individual consultation, respectively. In total, 286 patients got access to the website and were requested to visit the website. Seventy-seven patients of both groups actually logged-in on the website of which only 37 logged-in more than once. Since only a small proportion of the patients in group II and group III logged-in on their personalized website (34 and 43 patients for group II and group III, respectively), it was questionable whether there could be an effect of the website when compared to usual care. Therefore, we decided to report the results based on two groups, in which group I and group II combined were compared to group III, ie, we examined the effects of the group consultation plus the extra individual consultations.

From the 419 randomized patients, refill data of 260 patients were available. We used multiple imputation techniques for all missing data. See Figure 1.

\section{Results of the intervention}

\section{Baseline characteristics}

At baseline, the mean age of the participants was 61 years and $67 \%$ were male. Forty-seven percent of the participants were diagnosed with a stroke or TIA, 36\% with an acute coronary syndrome, and $17 \%$ with peripheral arterial disease. Ninety-eight percent received an antithrombotic agent and 94\% received lipid-lowering medication after the event. At baseline, MPR for all cardiovascular medication was $72 \%$. According to the MMS ${ }^{2}, 20 \%$ of all patients were low adherent, $46 \%$ and $35 \%$ were medium and high adherent, respectively. Mean NCD according to the BMQ was 3.6. Mean LDL was $2.5 \mathrm{mmol} / \mathrm{L}$ and mean systolic blood pressure was $137 \mathrm{mmHg}$. See Table 2 . 


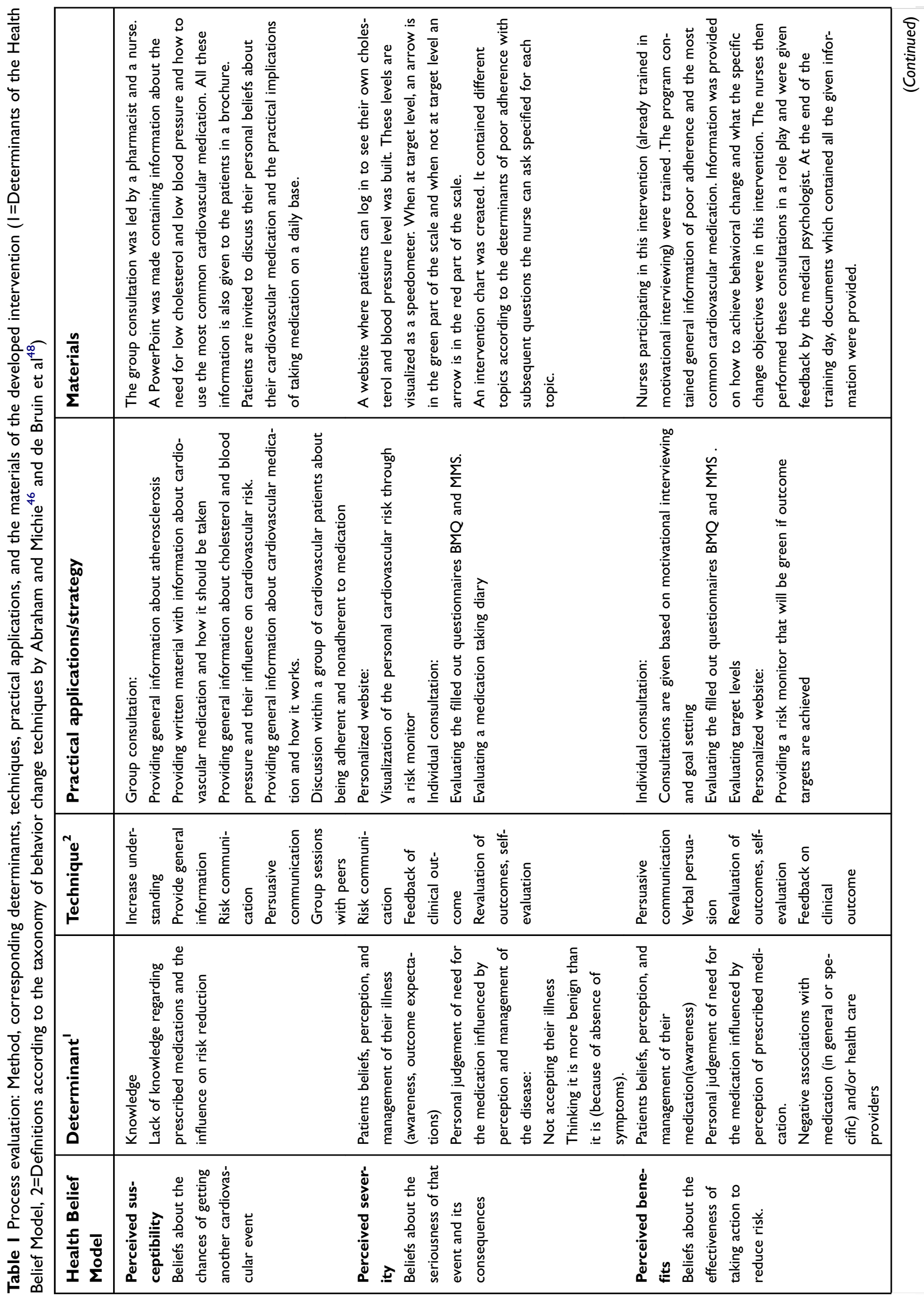




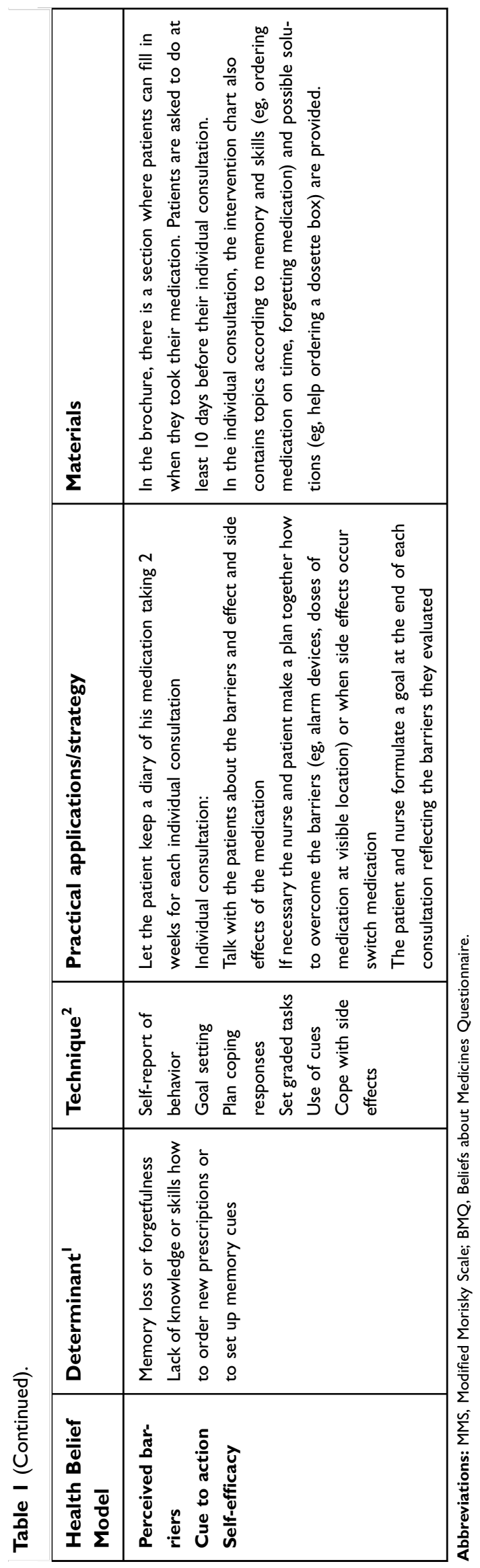

\section{Adherence}

The intervention did not show an effect on adherence to treatment. Patients in the usual care group had an adherence level of $93 \%$, compared to $89 \%$ in the intervention group ( $p$-value $=0.08)$ at $\mathrm{T} 2$. At T3, there also was no significant difference detected (adherence level was $81 \%$ and $76 \%$, respectively, for groups I and II with a $p$-value of 0.23 ). Percentage of adherent patients was $86.3 \%$ in the usual care group and $76.4 \%$ in the intervention group ( $p$-value $=0.17)$ at $\mathrm{T} 2$. This was $65 \%$ and $57.4 \%$ ( $p$-value $=0.38)$, respectively, at T3. Though we did not see differences in adherence between the usual care and intervention group, we observed a difference in adherence in time for both groups combined. Therefore, we performed a repeated measurement ANOVA in time without differentiating in trial groups. At $\mathrm{T} 1$, at $\mathrm{T} 2$, and at $\mathrm{T} 3$, the overall adherence was $72 \%, 92 \%$ and $80 \%$, respectively. During the study period, medication adherence increased with $20 \%$ (95\% CI $0.065-0.335)$. One year after this period, it declined with $12 \%(95 \%$ CI $0.073-0.17$ ).

\section{Clinical outcomes}

At T2, the mean LDL level was $2.2 \mathrm{mmol} / \mathrm{L}(2.5 \mathrm{mmol} / \mathrm{L}$ at baseline) and mean systolic blood pressure was 155 $\mathrm{mmHg}$ (136 mmHg at baseline) for both groups. There were no differences in LDL and blood pressure alteration between both groups. Blood pressure was above the target level in $76 \%$ of all patients and $12 \%$ showed a recorded LDL cholesterol level of $20 \%$ above the baseline LDL level ( $p$-value between groups was 0.52 and 0.4 , respectively).

\section{Patient outcomes}

No differences between the MMS ${ }^{\circledR}$ and the BMQ were detected at T2 between both groups. 29\% (usual care) and $31 \%$ (intervention group) of the patients showed a low adherence according to the MMS ${ }^{\circledR}$. There were no differences between the two groups studied ( $p$-value $=0.94)$. The mean NCD was 3.8 at T2. Again, no differences between the two studied groups were present (mean 3.8 vs mean 3.9, $p$-value $=0.86$ ). We did observe a difference in the four BMQ categories. In the intervention group, there was a shift from ambivalent (from $60 \%$ at $\mathrm{T} 1$ to $37 \%$ at $\mathrm{T} 2$ ) toward accepting (25\% at baseline to $33 \%$ at $\mathrm{T} 2$ ). In the usual care group, this shift was from ambivalent (63-43\%) to skeptical (4-15\%) and indifferent (6-15\%). 


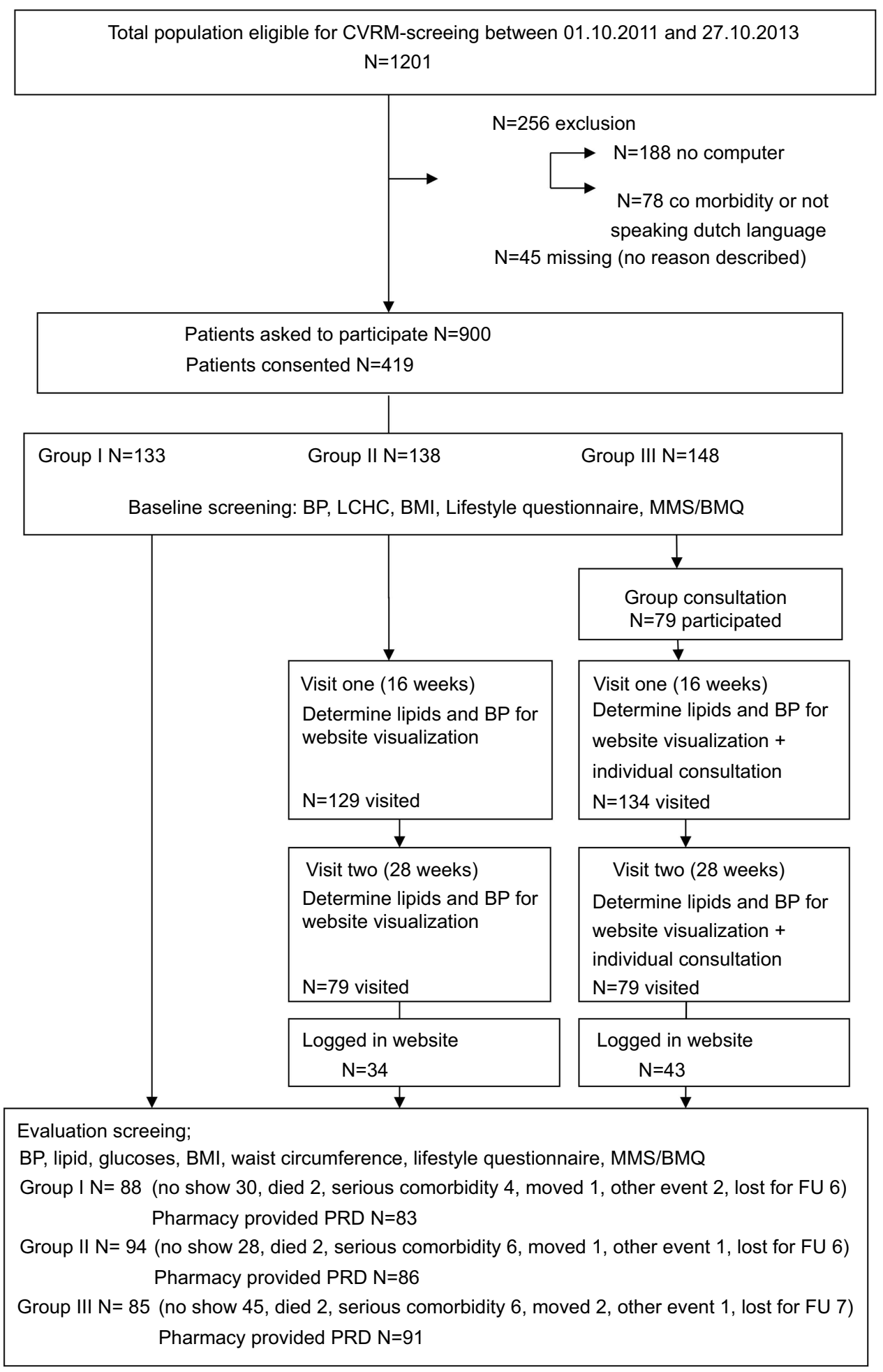

Figure I Patient enrollment and participation.

Note: LCHC depicts lipoprotein analysis with determination of high density lipoproteine-cholesterol and low density lipoproteine-cholesterol calculation.

Abbreviations: BMI, body mass index; PRD,pharmacy refill dates; FU, follow up; MMS, Modified Morisky Scale; BMQ, Beliefs about Medicines Questionnaire; CVRM, cardiovascular risk management; BP, blood pressure.

Differences in adherence, beliefs about medication, and clinical outcomes are depicted in Table 3A.
The observed adherence difference expressed in time is shown in Table 3B. 


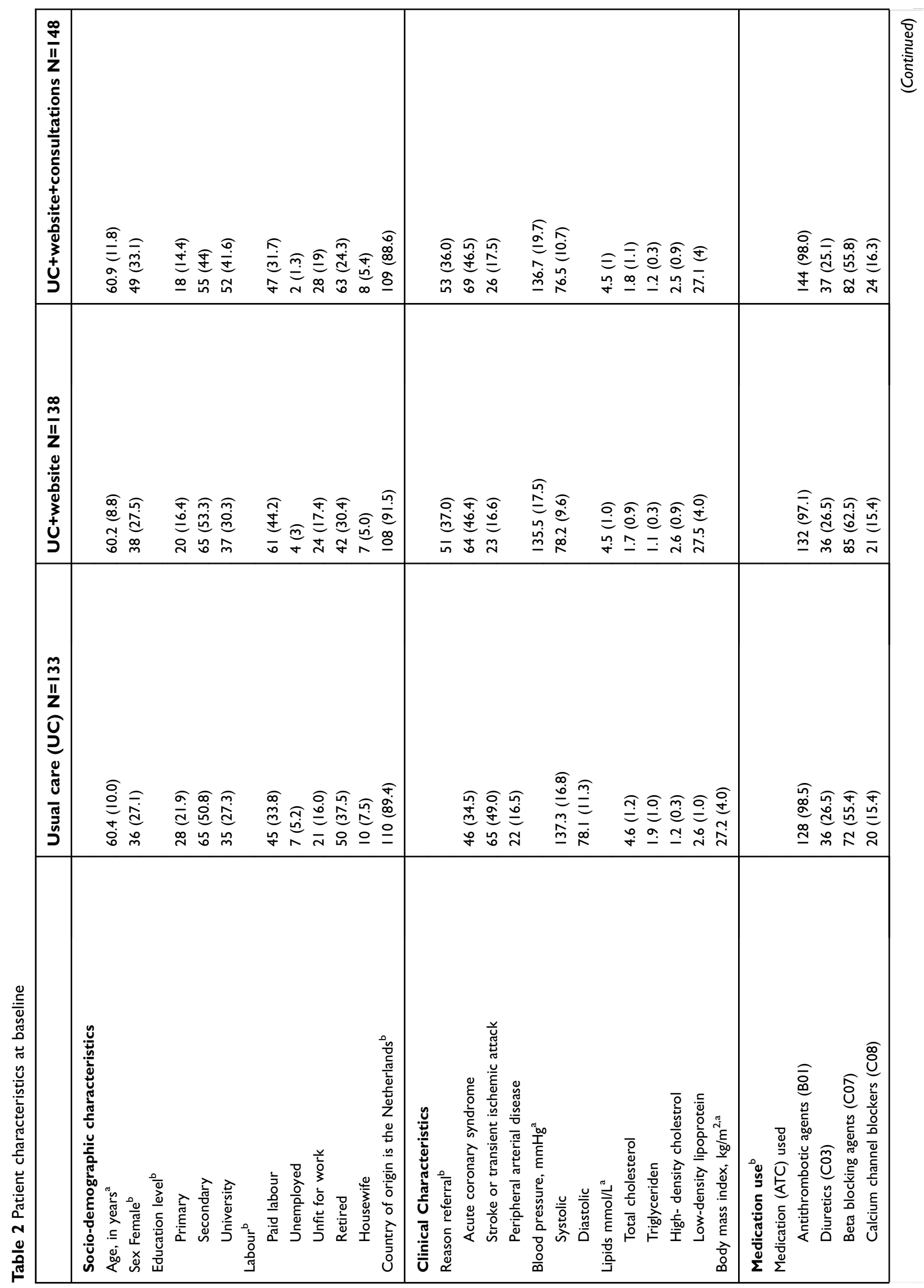




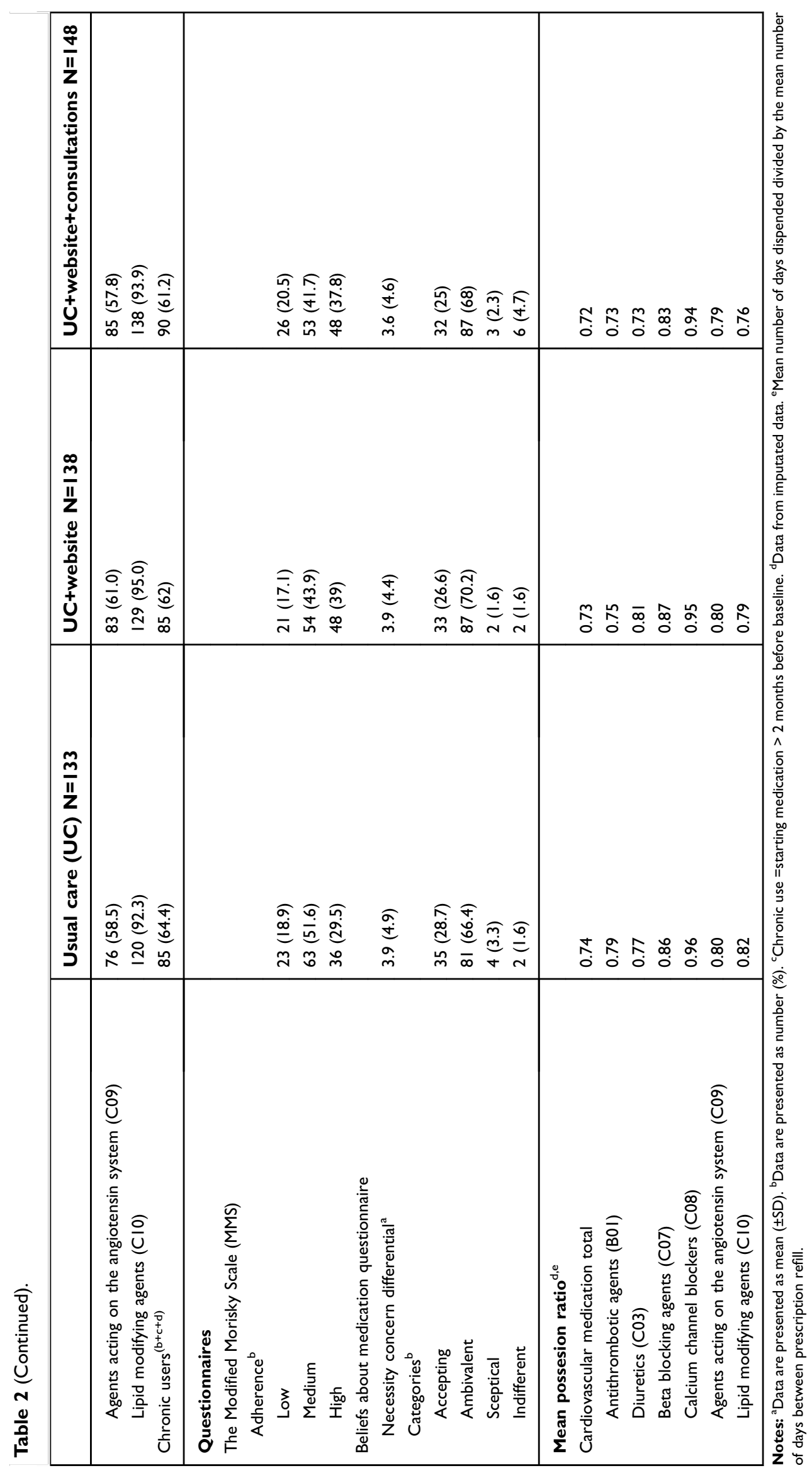


Table 3A Differences in medication adherence, beliefs about medication, and clinical outcome between usual care and intervention groups at baseline, after intervention and at follow-up of 12 months (all after imputation)

\begin{tabular}{|c|c|c|c|}
\hline & $\begin{array}{l}\text { Group I+II Usual care (UC) } \\
N=27 \mid\end{array}$ & $\begin{array}{l}\text { Group II UC+consultations } \\
N=\mid 48\end{array}$ & $p$-value ${ }^{a}$ \\
\hline \multicolumn{4}{|l|}{ Mean possesion ratio ${ }^{b}$} \\
\hline \multicolumn{4}{|l|}{ Total of cardiovascular medication } \\
\hline Baseline (TI) & 0.74 & 0.73 & \\
\hline After intervention (T2) & 0.93 & 0.89 & 0.08 \\
\hline Follow up of 12 months (T3) & 0.81 & 0.76 & 0.23 \\
\hline \multicolumn{4}{|l|}{ Per ATC-code } \\
\hline \multicolumn{4}{|l|}{ Baseline (TI) } \\
\hline Antithrombotic agents (BOI) & 0.77 & 0.73 & \\
\hline Diuretics (C03) & 0.79 & 0.73 & \\
\hline Beta blocking agents ( $\mathrm{C} 07)$ & 0.87 & 0.83 & \\
\hline Calcium channel blockers (C08) & 0.95 & 0.94 & \\
\hline Agents acting angiotensin (C09) & 0.80 & 0.79 & \\
\hline Lipid modifying agents $(\mathrm{ClO})$ & 0.80 & 0.76 & \\
\hline \multicolumn{4}{|l|}{ After intervention (T2) } \\
\hline Antithrombotic agents (BOI) & 0.92 & 0.91 & 0.65 \\
\hline Diuretics (C03) & 0.88 & 0.82 & 0.40 \\
\hline Beta blocking agents (C07) & 0.91 & 0.91 & 0.89 \\
\hline Calcium channel blockers (C08) & 0.89 & 0.87 & 0.57 \\
\hline Agents acting angiotensin (C09) & 0.92 & 0.91 & 0.56 \\
\hline Lipid modifying agents $(\mathrm{ClO})$ & 0.90 & 0.89 & 0.06 \\
\hline \multicolumn{4}{|l|}{ Follow up of 12 months (T3) } \\
\hline Antithrombotic agents (BOI) & 0.86 & 0.82 & 0.27 \\
\hline Diuretics (C03) & 0.83 & 0.76 & 0.12 \\
\hline Beta blocking agents (C07) & 0.80 & 0.80 & 0.87 \\
\hline Calcium channel blockers (C08) & 0.93 & 0.91 & 0.34 \\
\hline Agents acting angiotensin (C09) & 0.84 & 0.80 & 0.48 \\
\hline Lipid modifying agents $(\mathrm{Cl} 0)$ & 0.84 & 0.78 & 0.06 \\
\hline \multicolumn{4}{|l|}{ Adherent $^{c}$} \\
\hline Baseline (TI) & 53.5 & 48 & \\
\hline After intervention (T2) & 86.3 & 76.4 & 0.17 \\
\hline Follow up of 12 months (T3) & 64.9 & 76.4 & 0.38 \\
\hline \multicolumn{4}{|l|}{ Modified Morisky Scale $^{d}$} \\
\hline \multicolumn{4}{|l|}{ Baseline (TI) } \\
\hline Low & 19.6 & 21.6 & \\
\hline Medium & 46.5 & 41.2 & \\
\hline High & 32.7 & 37.2 & \\
\hline \multicolumn{4}{|l|}{ After intervention (T2) } \\
\hline Low & 28.7 & 31 & $0.94^{\mathrm{e}}$ \\
\hline Medium & 38 & 38.5 & \\
\hline High & 33.3 & 30.5 & \\
\hline \multicolumn{4}{|c|}{ Beliefs about medication questionnaire } \\
\hline \multicolumn{4}{|l|}{ Necessity concern differential ${ }^{f}$} \\
\hline Baseline (TI) & 3.7 & 3.4 & \\
\hline After intervention (T2) & 3.8 & 3.9 & 0.86 \\
\hline \multicolumn{4}{|l|}{ Categories $^{g}$} \\
\hline \multicolumn{4}{|l|}{ Baseline (TI) } \\
\hline Accepting & 27.3 & 25 & \\
\hline Ambivalent & 62.7 & 60.1 & \\
\hline
\end{tabular}

(Continued) 
Table 3A (Continued).

\begin{tabular}{|c|c|c|c|}
\hline & $\begin{array}{l}\text { Group I+II Usual care (UC) } \\
N=27 \mid\end{array}$ & $\begin{array}{l}\text { Group II UC+consultations } \\
N=\mid 48\end{array}$ & $p$-value ${ }^{a}$ \\
\hline Sceptical & 4.4 & 5.4 & \\
\hline Indifferent & 5.5 & 9.5 & \\
\hline \multicolumn{4}{|l|}{ After intervention (T2) } \\
\hline Accepting & 26.6 & 32.8 & \\
\hline Ambivalent & 43.2 & 37.1 & \\
\hline Sceptical & 14.8 & 17.6 & \\
\hline Indifferent & 15.2 & 12.6 & \\
\hline \multicolumn{4}{|l|}{ Clinical outcomes } \\
\hline \multicolumn{4}{|c|}{ Systolic blood pressure $(\mathrm{mmHg})$} \\
\hline Baseline (TI) & 136.4 & 136.7 & \\
\hline After intervention (T2) & 155 & 155 & 0.71 \\
\hline Adherent $^{\mathrm{h}}$ & 25 & 20.9 & 0.52 \\
\hline \multicolumn{4}{|c|}{ Low- density cholestrol (mmol/L) } \\
\hline Baseline (TI) & 2.5 & 2.5 & \\
\hline After intervention (T2) & 2.2 & 2.2 & 0.74 \\
\hline Adherent ${ }^{i}$ & 90.4 & 86.4 & 0.4 \\
\hline
\end{tabular}

Notes: ${ }^{a}$ Usual care and usual care + are combined and compared with usual care ++ group. ${ }^{b}$ Mean number of days dispended divided by the mean number of days between prescription refill. ' Percentage of patients (\%) considered adherent according to PRD total of the cardiovascular medication (PRD was above or equal to 0.8 ). ${ }^{\text {d Percentage of }}$ patients (\%) who had low, medium, or high adherent scores. ${ }^{\mathrm{e}}$ Low and medium adherence are combined and compared with high adherence group. ${ }^{\mathrm{f}} \mathrm{Differences}$ between concern and necessity scores (means). ' PPercentage of patients (\%) who are classified in the four different categories of the BMQ. ' $\mathrm{Percentage}$ of patients (\%) considered adherent according to systolic blood pressure ( $<135 \mathrm{mmHg}$ ) and percentage (\%) of patients using antihypertension drugs. 'Percentage of patients (\%) considered adherent according to LDL level (the recorded LDL level after intervention was $20 \%$ lower of baseline LDL cholesterol).

Table 3B Differences in mean medication adherence in time

\begin{tabular}{|l|l|l|l|}
\hline All participants $(\mathbf{N}=4$ I 9) & Baseline (TI) & After intervention (T2) & Follow-up I2 months (T3) \\
\hline $\begin{array}{l}\text { Mean possesion ratio } \\
\text { Total of cardiovascular medication }\end{array}$ & 0.72 & $0.92 *$ & $0.80^{* *}$ \\
\hline
\end{tabular}

Notes: ${ }^{*} p$-value $<0.05$ relative to TI. ${ }^{* *} p$-value $<0.05$ relative to $\mathrm{T} 2$.

\section{Discussion}

This study evaluated a nurse-led web-based intervention based on the $\mathrm{HBM}^{11,16,49}$ and the necessity-concern framework ${ }^{50}$ on medication adherence to treatment. Although we developed the intervention considering the recommendations of major reviews on medication adherence interventions, ${ }^{8,51-53}$ we did not see an effect of the developed intervention on our main adherence outcome. There are several explanations for the absence of an effect of this intervention. First, all patients in our study received the same structural cardiovascular care according to the European Guidelines of prevention of CVDs. $^{20}$ A previous evaluation of our cardiovascular screening program showed that a structural multidisciplinary evaluation and initiation of the best medical treatment in combination with addressing unhealthy lifestyle reduces cardiovascular risk as indicated by a reduction in smoking, alcohol consumption, unhealthy eating, blood pressure, and LDL-cholesterol level. ${ }^{54}$ Although medication adherence was not a structural approach in our usual care setting, the attention and screening on CVDrisk factors may have influenced adherence to medication adherence positively.

Second, in this study, we used pharmacy refill data as adherence measure. Refill adherence rates have extensively been used for the evaluation of medication adherence. Compared to electronic monitoring, refill data provide researchers with a relatively simple method for investigating adherence to medication in large populations. ${ }^{55}$ However, due to the increasing availability of automatic refills in the Netherlands, this measure may represent high adherence levels, while patients do not necessarily take their medication. ${ }^{10}$ By combining the refill data with a self-reported questionnaire, we, therefore, 
wanted to gain a fuller understanding of the adherence behavior of the patients. The results of the used questionnaire also showed no differences in medication adherence between the groups. Last, although the use of eHealth interventions is recommended to improve healthy behavior and for tailoring adherence interventions, ${ }^{44,56}$ the website was rarely used by our patients. Consequently, we were not able to study the perceived benefits of our eHealth intervention on adherence. Future studies should address this. There are several explanations as to why patients did not use the website. Patients had to use a special personal code and had to confirm their password with a code they received from a text message. These complicated steps may have influenced the use of the website negatively. High dropout rates may be a natural and typical feature of eHealth interventions, ${ }^{57}$ as was observed in our trial. There were fewer patients as expected who actually used the website and/or participated in the group consultation. This could be an indication that the intervention might be too intensive and experienced as a burden for patients. It could also mean that the intervention program was not enough exposed to enough patients to show a significant difference between the intervention group and the usual care group. We developed the intervention program by using constructs of the HBM and the necessity-concern framework but without involving patients. By personalizing the content of the individual consultations, we believed we tailored the intervention to the personal need of the patients. On the other hand, the process in which the content of the program was delivered was not tailored at all. Maybe only tailoring the content is not enough and the process in which the intervention is delivered also should be more tailored. For further research, we highly recommend involving patients in designing the intervention.

The study did show a difference in adherence in time for all groups. Adherence rates were higher during the study period and declined to levels comparable to baseline 1 year after the end of the study. Several explanations can be addressed for this phenomenon. First, participation in a study may have encouraged patients to be more adherent. ${ }^{9}$ Second, all patients who participated in this study recently had a cardiovascular event. For these patients, the need for adherent behavior is emerging. ${ }^{35,58}$ Yet, as the event fades and there are no more symptoms, adherence can also decline. ${ }^{2}$ This was also observed in our study. We looked even further to see if there was a difference in new users of CVRM medication and chronic users. We did observe a relatively high cohort of chronic users in our population, but this did not have any effect on the outcome. Although we did not establish a difference between groups in the NCD, we observed a shift in the categories from ambivalent toward accepting in the intervention group; this shift was not observed in the usual care group. In the ambivalent group, necessity beliefs are high but concern beliefs are also high. In the acceptance group, necessity beliefs are high but the concern beliefs are low. In order to know if the change in necessity category for the intervention group will have a positive effect on the adherence rate over time, we need to measure the adherence rate further in time.

Although overall adherence was relatively high at 12 months follow-up, only $20 \%$ of all patients had a systolic blood pressure within target. The mean blood pressure was even higher than it was at baseline. In contradiction, only $12 \%$ of all patients showed an LDL cholesterol level of $20 \%$ above the baseline LDL level. We cannot really explain this difference. Several major studies have demonstrated nonadherence to be an important cause for not reaching target levels, as well for lipid-lowering medication as for antihypertensive medication ${ }^{59,60}$ It can be suggested that not reaching the target level of systolic blood pressure is not necessarily due to nonadherence of the medication. ${ }^{61-63}$

\section{Limitations}

The study had several shortcomings. There were challenges with recruitment and due to an organizational decision to no longer supporting the website, we had to stop inclusion early and the original recruitment target was not met. This may have underpowered our results. However, the CI of the mean of the main outcome (the MPR) showed only a very small interval from -0.005 to 0.082 . Therefore, we may assume that a larger population size would not have made a difference in MPR between groups. We also had to deal with missing data, especially on the pharmacy refill data. The Dutch healthcare system does not provide a closed pharmacy system to a point of care (like a hospital). Therefore, we were dependent on the willingness of the pharmacists to provide us with refill data. This led to missing data. We also had a relatively high percentage of patients who did not complete the intervention and/or did not show up at the evaluation screening. By using multiple imputation golden standard for dealing with missing data, we believe we still provided a valid result of this study. ${ }^{38,39}$ Last, the nurses who performed the individual usual care consultations if needed (eg, to lose weight or to quit smoking) were the same nurses as the nurses who performed the consultations 
in our intervention group. This and the fact that all patients visited the same outpatient clinic while on chronic care support may lead to influencing among the groups. In order to prevent such contamination among the groups, the nurses who give the usual care should be different from those in the intervention groups.

\section{Conclusion}

Our intervention to improve medication adherence in cardiovascular patients did not show an effect on improving poor medication adherence. The intervention program was developed using the existing evidence and by applying this evidence. The intervention was also developed so it could easily be applied to the already existing structured usual care for secondary preventive cardiovascular care. By performing a process evaluation, we gained information that could help future researchers to include elements of this intervention. Elements of our intervention program could still lead to improving medication adherence, but we were not able to demonstrate it in this trial. This is maybe due to high adherence rates in both groups and/or the limited number of patients that complied with the intervention program. Adherence rates after the intervention were high in both the usual care and the intervention group. This could indicate that the structured provided care we already deliver to all cardiovascular patients has a positive effect on medication adherence. Or the effect of having a cardiovascular event was the key to better adherence.

\section{Acknowledgments}

This research received no specific grant from any funding agency in the public, commercial, or not-for-profit sectors.

\section{Disclosure}

The authors report no conflicts of interest in this work.

\section{References}

1. Piepoli MF, Hoes AW, Agewall S, et al. European guidelines on cardiovascular disease prevention in clinical practice: the sixth joint task force of the European society of cardiology and other societies on cardiovascular disease prevention in clinical practice (constituted by representatives of 10 societies and by invited experts) Developed with the special contribution of the European association for cardiovascular prevention \& rehabilitation (EACPR). Atherosclerosis. 2016;252:207-274. doi:10.1016/j.atherosclerosis.2016.05.037

2. Ho PM, Bryson CL, Rumsfeld JS. Medication adherence: its importance in cardiovascular outcomes. Circulation. 2009;119 (23):3028-3035. doi:10.1161/CIRCULATIONAHA.108.768986
3. Bansilal S, Castellano JM, Garrido E, et al. Assessing the impact of medication adherence on long-term cardiovascular outcomes. $\mathrm{J} \mathrm{Am}$ Coll Cardiol. 2016;68(8):789-801. doi:10.1016/j.jacc.2016.06.005

4. Hennein R, Hwang SJ, Au R, et al. Barriers to medication adherence and links to cardiovascular disease risk factor control: the Framingham heart study. Intern Med J. 2018;48(4):414-421. doi:10.1111/imj.13687

5. Sabate. Adherence to Long Term Therapies. Evedince for Action. Geneva: World Health Organization; 2003.

6. Valle JA, Ho PM. Medication adherence in secondary prevention post-myocardial infarction. Curr Treat Options Cardiovasc Med. 2014;16(12):349. doi:10.1007/s11936-014-0349-7

7. Chowdhury R, Khan H, Heydon E, et al. Adherence to cardiovascular therapy: a meta-analysis of prevalence and clinical consequences. Eur Heart J. 2013;34(38):2940-2948. doi:10.1093/eurheartj/eht295

8. Haynes RB, Ackloo E, Sahota N, McDonald HP, Yao X. Interventions for enhancing medication adherence. Cochrane Database Syst Rev. 2008;11(2):CD000011.

9. Horne R, Weinman J, Barber N, et al. Concordance, Adherence and Compliance in Medicine Taking. London: national Coordinating Centre for NHS Service and Organisation R\&D (NCCSDO); 2006.

10. Kronish IM, Ye S. Adherence to cardiovascular medications: lessons learned and future directions. Prog Cardiovasc Dis. 2013;55(6):590-600.

11. Hugtenburg JG, Timmers L, Elders PJ, Vervloet M, van Dijk L. Definitions, variants, and causes of nonadherence with medication: a challenge for tailored interventions. Patient Prefer Adherence. 2013;7:675-682. doi:10.2147/PPA.S29549

12. Kardas P, Lewek P, Matyjaszczyk M. Determinants of patient adherence: a review of systematic reviews. Front Pharmacol. 2013;4:91. doi:10.3389/fphar.2013.00091

13. Clifford S, Barber N, Horne R. Understanding different beliefs held by adherers, unintentional nonadherers, and intentional nonadherers: application of the necessity-concerns framework. J Psychosom Res. 2008;64(1):41-46. doi:10.1016/j.jpsychores.2007.05.004

14. Schedlbauer A, Davies P, Fahey T. Interventions to improve adherence to lipid lowering medication. Cochrane Database Syst Rev. 2010;(3):CD004371.

15. Petrilla AA, Benner JS, Battleman DS, Tierce JC, Hazard EH. Evidence-based interventions to improve patient compliance with antihypertensive and lipid-lowering medications. Int $J$ Clin Pract. 2005;59(12):1441-1451. doi:10.1111/j.1368-5031.2005.00704.x

16. Rosenstock IM, Strecher VJ, Becker MH. Social learning theory and the health belief model. Health Educ Behav. 1988;15(2):175-183.

17. Glanz K, Bishop DB. The role of behavioral science theory in development and implementation of public health interventions. In: Fielding JE, Brownson RC, Green LW, editors. Annual Review of Public Health, Vol. 31. Palo Alto: Annual Reviews; 2010:399-418.

18. Rosenstock IM. Historical origins of the Health Belief Model. Health Educ Behav. 1974;2(4):328-335.

19. Sieben A, van Onzenoort HA, van Laarhoven KJ, Bredie SJ, A Multifaceted nurse-. and web-based intervention for improving adherence to treatment in patients with cardiovascular disease: rationale and design of the MIRROR trial. JMIR Res Protoc. 2016;5(3): e187. doi:10.2196/resprot.5750

20. De Backer G, Ambrosioni E, Borch-Johnsen K, et al. European guidelines on cardiovascular disease prevention in clinical practice; third joint task force of European and other societies on cardiovascular disease prevention in clinical practice (constituted by representatives of eight societies and by invited experts). Eur J Cardiovasc Prev Rehabil. 2003;10(4):S1-S10.

21. Moore GF, Audrey S, Barker M, et al. Process evaluation of complex interventions: medical research council guidance. BMJ. 2015;350:h1258.

22. Hulscher ME, Laurant MG, Grol RP. Process evaluation on quality improvement interventions. Qual Saf Health Care. 2003;12(1):40-46. doi:10.1136/qhc.12.1.40 
23. Saunders RP, Evans MH, Joshi P. Developing a process-evaluation plan for assessing health promotion program implementation: a how-to guide. Health Promot Pract. 2005;6(2):134-147. doi:10.1177/1524839904273387

24. Oakley A, Strange V, Bonell C, Allen E, Stephenson J. Process evaluation in randomised controlled trials of complex interventions. BMJ. 2006;332(7538):413-416. doi:10.1136/bmj.332.7538.413

25. Cramer JA, Benedict A, Muszbek N, Keskinaslan A, Khan ZM. The significance of compliance and persistence in the treatment of diabetes, hypertension and dyslipidaemia: a review. Int J Clin Pract. 2008;62(1):76-87. doi:10.1111/j.1742-1241.2007.01630.x

26. O`Brien Eoin EE. European Guidelines on cardiovascular disease prevention in clinical practice (version 2012): the fifth joint task force of the European society of cardiology and other societies on cardiovascular disease Prevention in clinical practice (constituted by representatives of nine societies and by invited experts). Eur J Prev Cardiol. 2012;19(4):585-667. doi:10.1177/ 2047487312450228

27. Morisky DE, Ang A, Krousel-Wood M, Ward HJ. Predictive validity of a medication adherence measure in an outpatient setting. $J$ Clin Hypertens. 2008;10(5):348-354.

28. Krousel-Wood M, Islam T, Webber LS, Re RN, Morisky DE, Muntner P. New medication adherence scale versus pharmacy fill rates in seniors with hypertension. Am J Manag Care. 2009;15(1):59-66.

29. Morisky DE, DiMatteo MR. Improving the measurement of self-reported medication nonadherence: response to authors. J Clin Epidemiol. 2011;64(3):255-257; discussion 258-263. DOI:10.1016/j. jclinepi.2010.09.002

30. Horne R, Weinman J, Hankins M. The beliefs about medicines questionnaire: the development and evaluation of a new method for assessing the cognitive representation of medication. Psychol Health. 1999;14(1):1-24.

31. Horne R, Weinman J. Patients' beliefs about prescribed medicines and their role in adherence to treatment in chronic physical illness J Psychosom Res. 1999;47(6):555-567.

32. Menckeberg TT, Bouvy ML, Bracke M, et al. Beliefs about medicines predict refill adherence to inhaled corticosteroids. J Psychosom Res. 2008;64(1):47-54. doi:10.1016/j.jpsychores.2007.07.016

33. Aikens JE, Nease DE Jr., Nau DP, Klinkman MS, Schwenk TL. Adherence to maintenance-phase antidepressant medication as a function of patient beliefs about medication. Ann Fam Med. 2005;3(1):23-30. doi:10.1370/afm.238

34. Clatworthy J, Bowskill R, Parham R, Rank T, Scott J, Horne R. Understanding medication non-adherence in bipolar disorders using a necessity-concerns framework. J Affect Disord. 2009;116(1-2):51-55. doi:10.1016/j.jad.2008.11.004

35. Miller NH. Adherence behavior in the prevention and treatment of cardiovascular disease. J Cardiopulm Rehabil Prev. 2012;32 (2):63-70. doi:10.1097/HCR.1090b1013e318235c318729

36. Brown MT, Bussell JK. Medication adherence: WHO cares? Mayo Clin Proc. 2011;86(4):304-314. doi:10.4065/mcp.2010.0575

37. Blankers M, Koeter MW, Schippers GM. Missing data approaches in eHealth research: simulation study and a tutorial for nonmathematically inclined researchers. J Med Internet Res. 2010;12(5):e54. doi:10.2196/jmir.1587

38. Hayati Rezvan P, Lee KJ, Simpson JA. The rise of multiple imputation: a review of the reporting and implementation of the method in medical research. BMC Med Res Methodol. 2015;15:30. doi:10.1186/ s12874-015-0092-0

39. Plumpton CO, Morris T, Hughes DA, White IR. Multiple imputation of multiple multi-item scales when a full imputation model is infeasible. BMC Res Notes. 2016;9:45. doi:10.1186/s13104-0161938-1
40. Vink NM, Klungel OH, Stolk RP, Denig P. Comparison of various measures for assessing medication refill adherence using prescription data. Pharmacoepidemiol Drug Saf. 2009;18(2):159-165. doi:10.1002/ pds. 1698

41. Britt E, Hudson SM, Blampied NM. Motivational interviewing in health settings: a review. Patient Educ Couns. 2004;53(2):147-155. doi:10.1016/S0738-3991(03)00141-1

42. Linn AJ, van Weert JC, van Dijk L, Horne R, Smit EG. The value of nurses' tailored communication when discussing medicines: exploring the relationship between satisfaction, beliefs and adherence. $J$ Health Psychol. 2014;19:798-807.

43. Latter S, Maben J, Myall M, Young A. Perceptions and practice of concordance in nurses" prescribing consultations: findings from a national questionnaire survey and case studies of practice in England. Int $J$ Nurs Stud. 2007;44(1):9-18. doi:10.1016/j. ijnurstu.2005.11.005

44. Barak A, Klein B, Proudfoot J. Defining internet-supported therapeutic interventions. Ann Behav Sci Med Educ. 2009;38(1):4-17. doi:10.1007/s12160-009-9130-7

45. Alpay LL, Henkemans OB, Otten W, Rovekamp TA, Dumay AC. E-health applications and services for patient empowerment: directions for best practices in The Netherlands. Telemed $J$ E Health. 2010;16(7):787-791. doi:10.1089/tmj.2009.0156

46. Abraham C, Michie S. A taxonomy of behavior change techniques used in interventions. Health Psychol. 2008;27(3):379-387. doi:10.1037/0278-6133.27.3.379

47. Michie S, Prestwich A. Are interventions theory-based? Development of a theory coding scheme. Health Psychol. 2010;29 (1):1-8. doi:10.1037/a0016939

48. de Bruin M, Viechtbauer W, Schaalma HP, Kok G, Abraham C, Hospers HJ. Standard care impact on effects of highly active antiretroviral therapy adherence interventions: a meta-analysis of randomized controlled trials. Arch Intern Med. 2010;170(3):240-250. doi:10.1001/archinternmed.2009.536

49. Ryan R, Santesso N, Hill S, Lowe D, Kaufman C, Grimshaw J. Consumer-oriented interventions for evidence-based prescribing and medicines use: an overview of systematic reviews. Cochrane Database Syst Rev. 2011;(5):CD007768.

50. Horne R, Chapman SC, Parham R, Freemantle N, Forbes A, Cooper V. Understanding patients" adherence-related beliefs about medicines prescribed for long-term conditions: a metaanalytic review of the necessity-concerns framework. PLoS One. 2013;8(12):e80633. doi:10.1371/journal.pone. 0080633

51. Brian Haynes R, Ann McKibbon K, Kanani R. Systematic review of randomised trials of interventions to assist patients to follow prescriptions for medications. Lancet. 1996;348(9024):383-386. doi:10.1016/S0140-6736(96)01073-2

52. Haynes RB, McDonald HP, Garg AX. Helping patients follow prescribed treatment. JAMA. 2002;288(22):2880-2883. doi:10.1001/ jama.288.22.2880

53. McDonald H, Garg AX, Haynes RB. Interventions to enhance patient adherence to medication prescriptions: scientific review. JAMA. 2002;288(22):2868-2879. doi:10.1001/jama.288.22.2868

54. van Den Wijngaart LS, Sieben A, van der Vlugt M, de Leeuw FE, Bredie SJ. A nurse-led multidisciplinary intervention to improve cardiovascular disease profile of patients. West J Nurs Res. 2015;37 (6):705-723. doi:10.1177/0193945914533427

55. Krousel-Wood MA, Muntner P, Islam T, Morisky DE, Webber LS. Barriers to and determinants of medication adherence in hypertension management: perspective of the cohort study of medication adherence among older adults. Med Clin North Am. 2009;93(3):753-769. doi:10.1016/j.mcna.2009.02.007 
56. Linn JA, Vervloet M, van Dijk L, Smit GE, Van Weert CMJ. Effects of eHealth interventions on medication adherence: a systematic review of the literature. $J$ Med Internet Res. 2011;13(4):e103. doi:10.2196/jmir.1658

57. Eysenbach G. The law of attrition. J Med Internet Res. 2005;7(1): e11. doi:10.2196/jmir.7.5.e60

58. Jackevicius CA, Li P, Tu JV. Prevalence, predictors, and outcomes of primary nonadherence after acute myocardial infarction. Circulation. 2008;117(8):1028-1036. doi:10.1161/CIRCULATIONAHA.107.706820

59. Naderi SH, Bestwick JP, Wald DS. Adherence to drugs that prevent cardiovascular disease: meta-analysis on 376,162 patients. Am J Med. 2012;125(9):882-887.e881. doi:10.1016/j. amjmed.2011.12.013
60. Simpson RJ Jr., Mendys P. The effects of adherence and persistence on clinical outcomes in patients treated with statins: a systematic review. J Clin Lipidol. 2010;4(6):462-471. doi:10.1016/j.jacl.2010.08.026

61. van Onzenoort HA, Menger FE, Neef C, et al. Participation in a clinical trial enhances adherence and persistence to treatment: a retrospective cohort study. Hypertension. 2011;58(4):573-578. doi:10.1161/HYPERTENSIONAHA.111.171074

62. Wetzels GE, Nelemans P, Schouten JS, Prins MH. Facts and fiction of poor compliance as a cause of inadequate blood pressure control: a systematic review. J Hypertens. 2004;22(10):1849-1855.

63. Horne R, Clatworthy J, Hankins M. High adherence and concordance within a clinical trial of antihypertensives. Chronic Illn. 2010;6 (4):243-251. doi:10.1177/1742395310369018

\section{Publish your work in this journal}

Patient Preference and Adherence is an international, peer-reviewed, open access journal that focusing on the growing importance of patient preference and adherence throughout the therapeutic continuum. Patient satisfaction, acceptability, quality of life, compliance, persistence and their role in developing new therapeutic modalities and compounds to optimize clinical outcomes for existing disease states are major areas of interest for the journal. This journal has been accepted for indexing on PubMed Central. The manuscript management system is completely online and includes a very quick and fair peer-review system, which is all easy to use. Visit http:// www.dovepress.com/testimonials.php to read real quotes from published authors. 